\title{
There is no evidence that associations between adolescents' digital tech- nology engagement and mental health problems have increased
}

\author{
Matti Vuorre ${ }^{1}$, Amy Orben $^{3,4}, \&$ Andrew K. Przybylski ${ }^{1,2}$ \\ ${ }^{1}$ Oxford Internet Institute, University of Oxford, ${ }^{2}$ Department of Experimental Psychol- \\ ogy, University of Oxford, ${ }^{3}$ MRC Cognition and Brain Sciences Unit, University of \\ Cambridge, ${ }^{4}$ Emmanuel College, University of Cambridge
}

\begin{abstract}
Digital technology is ubiquitous in modern adolescence, and researchers are concerned that it has negative impacts on mental health that, furthermore, increase over time. To investigate if technology is becoming more harmful, we examined changes in associations between technology engagement and mental health in three nationally representative samples. Results were mixed across types of technology and mental health outcomes: Technology engagement had become less strongly associated with depression in the past decade, but social media use more strongly associated with emotional problems. We detected no changes in five other associations, or differential associations by gender. There is therefore little evidence for increases in the associations between adolescents' technology engagement and mental health. Information about new digital media has been collected for a relatively short time; drawing firm conclusions about changes in their associations with mental health may be premature. We urge transparent and credible collaborations between scientists and technology companies.
\end{abstract}

Keywords: Mental health; Depression; Social media; Adolescents

Any understanding of 21 st century adolescence would be incomplete without an appreciation of social media platforms and other digital technologies, which have become an integral element of young people's everyday lives over the last decades (Anderson \& Jiang, 2018; Taylor \& Silver, 2019). Accompanying this growth in popularity of digital technologies, downward trends in adolescent mental health have been observed in certain countries (Centers for Disease Control and Prevention, 2020). Although there is a large body of correlational and observational research, some of it

Manuscript in press at Clinical Psychological Science.

This research was supported by the Huo Family Foundation and the Economic and Social Research Council (ES/T008709/1). Monitoring the Future is supported by the National Institute on Drug Abuse; Understanding Society is sponsored by the Economic and Social Research Council; YRBS is conducted and funded by the Centers for Disease Control and Prevention. The authors thank Niklas Johannes, Chris Ferguson, and an anonymous reviewer for comments on an earlier draft of this manuscript. All three authors have had full access to the data and MV takes responsibility for its integrity and the accuracy of the data analysis. AKP had final responsibility for the decision to submit for publication. highly visible, into the association, there is little good evidence that technology use causes decreases in mental health (Dickson et al., 2019). Nevertheless, worries about the potential negative effects of technology have emerged as a dominant topic in popular discussion (Etchells et al., 2016; Jensen et al., 2019; Kardefelt-Winther et al., 2020; Orben et al., 2019; Orben \& Przybylski, 2019a; Twenge et al., 2017).

However, concerns about digital technologies are best understood to follow a pattern of societal apprehension that waxes and wanes as novel forms of entertainment and social technologies are invented and gradually adopted by young people (Orben, 2020). While parents and scholars are currently concerned about the impact of social media and digital technologies such as smartphones on mental health, the early twentieth century saw dime novels blamed for eliciting mania and risk taking (Furedi, 2015). A generation later, the immersive nature of radio dramas was thought to make young listeners vulnerable to ill health, sleep loss, and anxiety (Preston, 1941). These concerns were largely forgotten by mid-century when the visually stimulating characteristics of comic books and television were implicated as factors predisposing adolescents to maladjustment and antisocial behaviour (Wertham, 1954). Most recently, video games were suggested to produce a generation of violent criminals (Bushman \& Anderson, 2002), but this argument has since 
been refuted (Drummond et al., 2020; Ferguson et al., 2020). Within this cycle, novel technologies spur research that focuses on their distinctive new features, leading to researchers overlooking time-dependent changes within technology's associations with mental health and thus treating them as fixed.

Given this, a more accurate understanding of how children and young people are affected by new technologies would be enabled by studying their effects over time. But because researchers have assumed that technology effects are fixed, at least two time-dependent changes have evaded systematic testing. First, when fears emerge about a new technology, worries about previous technologies are largely abandoned without an agreement on —or good data indicating - whether, why or how the previous technologies were or were not harmful (Grimes et al., 2008; Orben, 2020). In a stepwise fashion, focus is instead redirected to the new technology of concern with the suggestion that it is more harmful due to technological advancement. Because interest moves elsewhere, there are few or no tests of whether the effects of previous technologies, such as those of watching television, actually increased or decreased as society's attention shifted to the possible harms associated with more recent technologies, e.g. playing video games. Therefore, we have not learned how existing technologies' effects change as new technologies emerge and are adopted by young people.

Second, some technologies might have an increasingly harmful influence on adolescent mental health by virtue of their dynamic nature. When a new technology reaches a threshold of popularity in society, a constellation of distinctive features, such as portability, immersiveness, or online connectivity, are often highlighted to explain why society should become worried about this new innovation over and above previous ones. Yet little attention is paid to how a given technology's effects might shift over time, as affordances expand, or the underlying business models drive designers to change how adolescents use them. This is especially the case with newer digital technologies like social media platforms, whose underlying algorithms are continuously optimised to capture attention, data, and drive engagement through alterations in design, novel features or apps (Lewis, 2017; Solon, 2017). Therefore, it remains a reasonable, yet untested, assumption that their possible negative influence on mental health could be magnified over time by virtue of their dynamic nature.

\section{Understanding Digital Harm}

In the present study we addressed these shortcomings by investigating how the associations between digital technology use and adolescent mental health vary over time. First, we examined the possible declines in observed relationships between television, a technology for which public concern has largely shifted away from in recent years, and mental health. Second, we investigated potential increases in negative associations between mental health, and social media and digital devices, technologies which, we have good reason to think, might have increased in harmfulness recently. To provide context for understanding changing associations over time, we first discuss the evidence supporting the idea that digital technologies promote harm in the first place.

Currently, the literature suggesting a negative link between digital technology engagement and mental health is decidedly mixed and grounded on suboptimal methodologies and data (Dickson et al., 2019; Odgers \& Jensen, 2020). That is, the observed associations are typically based on between-persons correlations, which make determining causality and directionality difficult, and are susceptible to the influence of confounding observed or unobserved variables (Ophir et al., 2020). Literature reviews by academic, medical, and policy collaborations have highlighted both the lowquality evidence in this space and the need for a longitudinal perspective (Appel et al., 2020; Hawkes, 2019; Viner et al., 2019). In secondary data analyses, these problems are compounded by a general lack of adherence to consistent standards (Weston et al., 2019), which threatens the validity and robustness of reported associations, as the many choices leading to a reported analysis can engender misrepresentative estimates (Orben \& Przybylski, 2019a). Studies that have adopted more systematic approaches to estimating associations between well-being and technology engagement have shown that the resulting relations depend on gender, arbitrary analytic choices, and the exact variables included in the analyses (Bjerre-Nielsen et al., 2020; Jensen et al., 2019; Kreski et al., 2020; Orben et al., 2019). In addition, most of this literature is limited by its use of self-reports of technology use, which are known to be biased and noisy indicators of true amounts of engagement, and lead to inaccurate estimated relations between health and well-being (Johannes et al., 2020; Parry et al., 2020; Scharkow, 2016; Shaw et al., 2020). Nevertheless, recent analyses and reviews have shown a trend whereby individuals who report greater technology engagement tend to also report higher levels of mental health problems, but the factors underlying these cross-sectional relationships still remain poorly understood (Appel et al., 2020).

\section{Digital Dynamics}

One of the reasons for the lack of consensus around ideas such as digital or online harms might be that studies investi- 
gating it do not regularly consider the possibility that technologies' impacts on young people are likely to change over time as the technologies, and ways in which they are used, change. Technologies are often considered as unitary causes of change in society, through a process called technological determinism, and academics therefore rarely treat them as dynamic when trying to understand their effects on young and vulnerable people (Grimes et al., 2008; Orben, 2020). However, technologies, and their uses, are not static. The contexts and ways in which digital technologies are used are themselves in flux, with different platforms and technologies gaining and losing popularity within an adolescent's social group (Boyd, 2014). These shifts have the potential to shape differences over time in user motivations and the associations between technology engagement and mental health, and could therefore cause certain technologies' effects on mental health to increase or decrease over long periods of time.

Furthermore, not only do the contexts of use change, but the technologies themselves are developing. This is particularly true for video gaming and online platforms which are, essentially, continuously updating virtual social hubs. New business and monetisation models, features, play modes, and social trends emerge regularly. In other words, material changes in how technologies affect individuals and societies may therefore not be marked by an obvious stepwise shift between two successive forms of technology, say from radio to television. Instead, more subtle advances within a single technological milieu might drive profound changes. For example, the addition of so called dark patterns - features that are designed to manipulate users to e.g. purchase items (Singer, 2016) - and attention demanding features that undermine users' psychological autonomy could serve to magnify negative impacts of social media, whereas tools that empower or connect users in new ways could do the opposite and promote health and well-being (Lewis, 2017; Solon, 2017; Verduyn et al., 2017). In the same vein, observed differences might arise between demographic cohorts, such as genders and age groups, might arise because of algorithmically driven market segmentation of online platforms which are more negatively influential to certain young populations' mental health. One worry, then, is that these technologies are advancing "behind the scenes" and becoming more harmful to adolescent health without parents or society being aware of any obvious changes.

Although the possibility that digital technologies' effects on adolescents are not static would have substantial implications for regulation, and this potential is often discussed in public conversation, the idea has garnered only limited attention in empirical studies which have typically treated the relation as invariant over time (but see Ferguson, 2020; Kreski et al., 2020). The current study therefore aims to provide one of the first large-scale examinations into whether the associations between adolescent technology use and mental health shift over time.

\section{The Current Study}

To probe whether the associations between technology engagement and mental health have indeed changed over time, a comprehensive perspective is needed. In the current study we therefore not only investigate the use of smartphones and social media but also that of television use, which elicited comparable levels of academic, public and policy concern about its potential impact on child development in the mid and late 20th century (Orben, 2020). While discussions surrounding television's purported harm have largely receded, research never conclusively demonstrated that it ceased being harmful, or that the fears were not well founded in the first place. The long timeframe of data available regarding television viewing provides a baseline for testing its relations with health outcomes over time to determine if its associations with mental health did wane as society's and researchers' attention was refocused to newer forms of technology. Further, it provides a point of empirical contrast to contextualise the extent to which shifts in the associations relating digital devices and social media use to mental health follow the same pattern or have become more negative in the shorter periods of time that they have been used and for which there is data.

The current study therefore analysed data from three large-scale studies of adolescents living in the United States (Monitoring the Future, MTF; Youth Risk Behavior Surveillance System, YRBS) and the United Kingdom (Understanding Society; US) to probe how the associations between adolescent mental health and use of various technologies had changed over time. Our primary interest was determining whether the relationship between technology use and adolescent mental health had changed over different timespans. As a supplementary analysis, because previous literature has highlighted important differences between boys and girls in novel technologies' associations with adolescent mental health (Kelly et al., 2018; Kreski et al., 2020), we also investigated the moderating role of sex.

\section{Methods}

\section{Study design and participants}

We focused on three large nationally representative datasets, from the United States and United Kingdom, with 
pertinent variables on adolescent mental health and technology engagement, and a combined total of 430,561 participants. Monitoring the Future (MTF) is an annual survey of $8^{\text {th }}, 10^{\text {th }}$, and $12^{\text {th }}$ graders in the US, and has been administered to $8^{\text {th }}$ and $10^{\text {th }}$ graders since 1991 by The University of Michigan Institute for Social Research (Miech et al., 2020). Understanding Society (US) is a longitudinal survey of approximately 40,000 households in the UK, and has been conducted since 2009 by the Institute for Social and Economic Research at the University of Essex (University of Essex, Institute for Social and Economic Research., 2019). Youth Risk Behavior Surveillance System (YRBS) is a biennial survey of $9^{\text {th }}$ to $12^{\text {th }}$ graders in the US, and has been conducted since 1991 by the CDC (Kann, 2018).

Although all these studies also included older participants, we particularly focused on adolescents, and thus restricted the analyses to individuals aged between 10 and 15 years (MTF does not include participant age, but the $8^{\text {th }}$ and $10^{\text {th }}$ graders in those data are likely to fall within this age range).

\section{Outcomes}

The surveys, particularly MTF and US, include a variety of health and well-being measures ranging from subjective well-being, such as loneliness and self-esteem, to constructs that are typically thought to indicate more objective mental health problems, such as depression and suicidality. Because our focus was solely on mental health rather than subjective well-being, we narrowed our investigation to the four mental health outcomes detailed below.

Depression was measured in the MTF dataset with six items that have been used in previous research (Miech et al., 2020; Newcomb et al., 1981; Twenge et al., 2017), although not always in a consistent manner (Orben \& Przybylski, 2019a, see especially supplementary table 6). Respondents rated their agreement to each item (e.g. "Life often seems meaningless") on a 5-point scale from "Disagree" to "Agree". 380,924 $8^{\text {th }}$ and $10^{\text {th }}$ graders responded to at least one item on the scale, whose items provided good reliability $($ alpha $=.85)$. We averaged these items to a mean score for each individual.

US queried for externalising and internalising mental health problems with the five-item conduct (e.g. "I get very

1 The fourth item asked about the number of suicide attempts, but we converted it to a yes/no indicator, a decision justified by low cell counts in the higher categories. A fifth item queried the severity of suicide attempts, but we did not use this item, because responses to it were conditional on positive response to the attempts question. angry and often lose my temper") and emotional (e.g. "I worry a lot") problems subscales of the Strengths and Difficulties Questionnaire (Goodman et al., 2000). 19,190 (conduct) and 19,184 (emotional problems) 10 to 15-year olds rated their agreement on a three-point scale from "Not true" to "Certainly true" to at least one of these items, which indicated moderate reliabilities (alphas .62 and .69, respectively). We averaged the items in each subscale to assign two mean scores for each individual. Outcomes in the MTF and US datasets showed low item-level missingness (complete responses: $\mathrm{MTF}=95 \%$, US $=96 \%$ ); missing item values were ignored in computing the scale means.

YRBS measured depressive mood and suicidal ideation and behaviour with five items (e.g. "During the past 12 months, did you ever feel so sad or hopeless almost every day for two weeks or more in a row that you stopped doing some usual activities?", and "During the past 12 months, did you ever seriously consider attempting suicide?") to which participants provided binary yes/no responses. ${ }^{1} 30,30512$ to 15-year old individuals answered one or more of these items. Due to the heterogeneity between these items, we did not average across them, but instead opted for a modelling strategy that accounted for variability between items (see 'Statistical analysis').

\section{Explanatory variables}

All three datasets asked participants to estimate their average weekday TV viewing hours. Social media use was queried in $\mathrm{MTF}^{2}$ ("How often do you [...] Visit social networking websites (like Facebook)"; 1 = "Never" to 5 = "Almost every day"; 120,265 respondents) and US ("Hours using social media on weekdays"; 1 = "None" to $5=$ "7 or more hours"; 18,999 respondents). YRBS asked about daily digital device use ("On an average school day, how many hours do you play video or computer games or use a computer for something that is not school work? (Count time spent on things such as Xbox, PlayStation, an iPad or other tablet, a smartphone, texting, YouTube, Instagram, Facebook, or other social media.)"; 1 = "I do not play video or computer games or use a computer for something that is not school work" to $7=$ " 5 or more hours per day"; 29,672 respondents). All datasets included variables for the year in which the data was collected, ${ }^{3}$ and the respondents' gender. Rows

2 There were small variations in some questions over time, such as the MTF social media item, which included MySpace as an example before 2010 .

${ }^{3}$ US data includes the data collection wave, but not the year in which the data was collected. Because the US data collection for each wave takes place over a 2-year period, 
of data where the sex variable was missing were excluded (MTF: $4 \%$, US: $0.02 \%$, YRBS: $0.3 \%$ ) to allow using sex as a moderator in our analyses below.

\section{Statistical analysis}

Our main goal was to investigate how associations between adolescents' technology use and mental health had changed over time. We adopted the simplifying assumption that any change to that relationship had been linear over the time frame studied. That is, we investigated whether there had been overall increases or decreases in the associations. To do this, we estimated eight models, one for each outcome-predictor pair in a given dataset. We modelled each outcome (e.g. depression; standardized) on year (centered on 2017, the most recent year for which data was available), the explanatory variable (e.g. TV use; standardized), and their interaction. Additionally, sex (contrast coded as $-0.5=$ male, $0.5=$ female) and its interactions with other predictors was included to investigate its moderating roles, particularly of the yearly changes in technology effects. For analyses of the US data, which is longitudinal unlike the other two datasets, we assigned random intercepts for individuals (Gelman \& Hill, 2007), and used between-person specific predictorsi.e. the person-specific means of the grand-mean deviated predictors - to separate between-person variation, the target of this study, from within-person variation. Outcomes in the MTF and US analyses were treated as normally distributed.

We modelled the binary YRBS item responses as Bernoulli distributed with a probit link function and specified all parameters as random across items. This modelling strategy had two advantages over standard gaussian analyses of either the individual items or their averages: First, the item responses are not normally distributed, and assuming so would risk distorted effect size estimates. Second, analysing the items separately would risk spurious findings due to mul- tiple comparisons, but aggregating them would falsely ignore their heterogeneity (e.g. one item measured suicidal behaviour, which is less prevalent than anhedonia). Instead, this hierarchical modelling strategy allowed for differences between items, while also partially pooling information across them and allowing inferences about their average, which we call suicidal ideation and behaviour, below (Gelman \& Hill, 2007). Similar to models of the US and MTF datasets, we ignored missing item-level values. All models were estimated within the Bayesian framework using minimally informative priors (see online analysis supplement); we report posterior means and quantiles as parameters' point estimates and credibility intervals (CI) (Bürkner, 2017; Carpenter et al., 2017).

\section{Data and code availability}

All annotated study code is available at https://digitalwellbeing.github.io/timetrends and archived at https:/osf.io/4zat9/. The data are available at https://www.cdc.gov/healthyyouth/data/yrbs/data.htm (YRBS), https://www.understandingsociety.ac.uk/documentation/access-data (US), and https://www.icpsr.umich.edu/icpsrweb/ICPSR/series/35 (MTF).

\section{Results}

Before modelling the technology-mental health relations, and their changes over time, we estimated Pearson's $r$ correlations between the technology metrics and mental health outcomes separately for each year, to illustrate the year-specific technology correlations without assuming linear change in them over time. These cross-sectional between-person relationships are shown in Figure 1, which suggested that changes in the relationship over time were plausible, but likely to differ between outcomes and technologies. we converted waves to years using the year in which that wave's data collection began. 


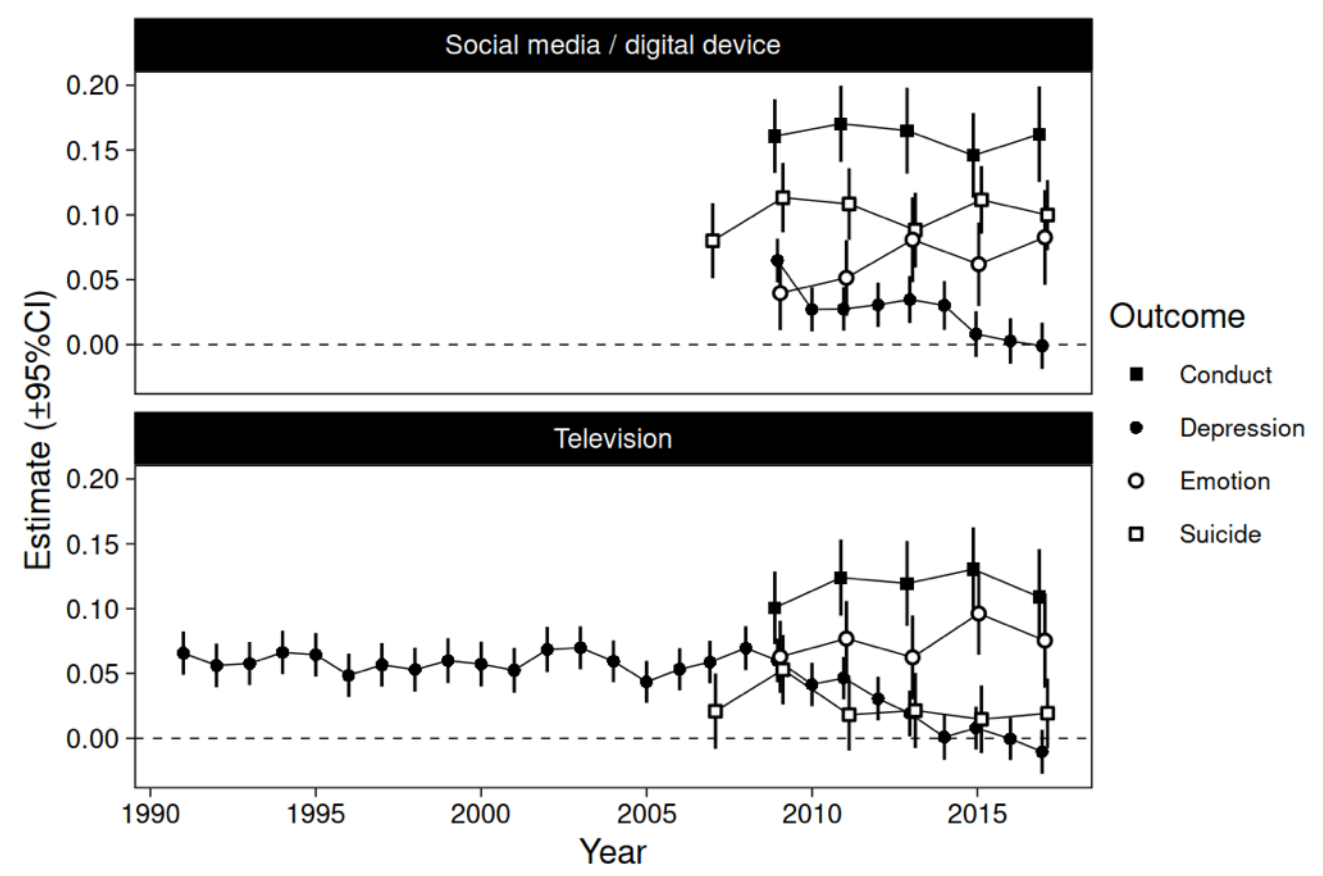

Figure 1. Associations between adolescents' technology use and mental health outcomes, estimated separately for each year with Pearson's $r$. Error bars indicate 95\% CIs. "Suicide" indicates the mean of the YRBS suicidal ideation and behaviour items. The points are horizontally jittered to display overlapping estimates.

We continued our analyses by estimating our models of interest. We first focused on the parameters indexing the association between technology use and mental health in 2017 (analogous to the rightmost points in Figure 1): There were notable differences in associations across the different mental health outcomes and technologies (Figure 2, top panel). These parameters indicate the relationship between a given technology and mental health outcome in the year 2017, because year was included in the model and centered at 2017. The parameter magnitudes are in standard deviations, because we standardized the variables or, in the case of suicidality, used a probit link function, which is equivalent to assuming a standard normal latent variable underlying the binary responses. Conduct problems were positively related to both TV and social media, whereas depression's relation to both was practically zero. Emotional problems were also related to both technologies, but suicidal ideation and behaviour was reliably associated only with social media. The magnitudes of these relations were within the ranges of previous findings (that have mainly ignored differences between years), and are generally considered to be very small.
Most importantly, regarding our main research question, the interaction parameters indexing the change in technology-mental health associations over time are shown in the bottom panel of Figure 2. The parameters indicate the magnitude by which each technology relationship either increased (positive) or decreased (negative values) in one year. The relationship between social media and $\mathrm{TV}$, and conduct problems had remained stable, as indicated by the estimated parameters narrowly centered on zero. Depression's association with social media and TV had decreased over time. The association between emotional problems and social media had increased, but their association with TV had remained stable. Interestingly, no credible changes were detected in the relationship between suicidal ideation and behaviour and either social media or television. Notably, even the greatest magnitudes of these parameters did not exceed 0.01 change in standard deviation units, suggesting that changes, if any, were very small. 


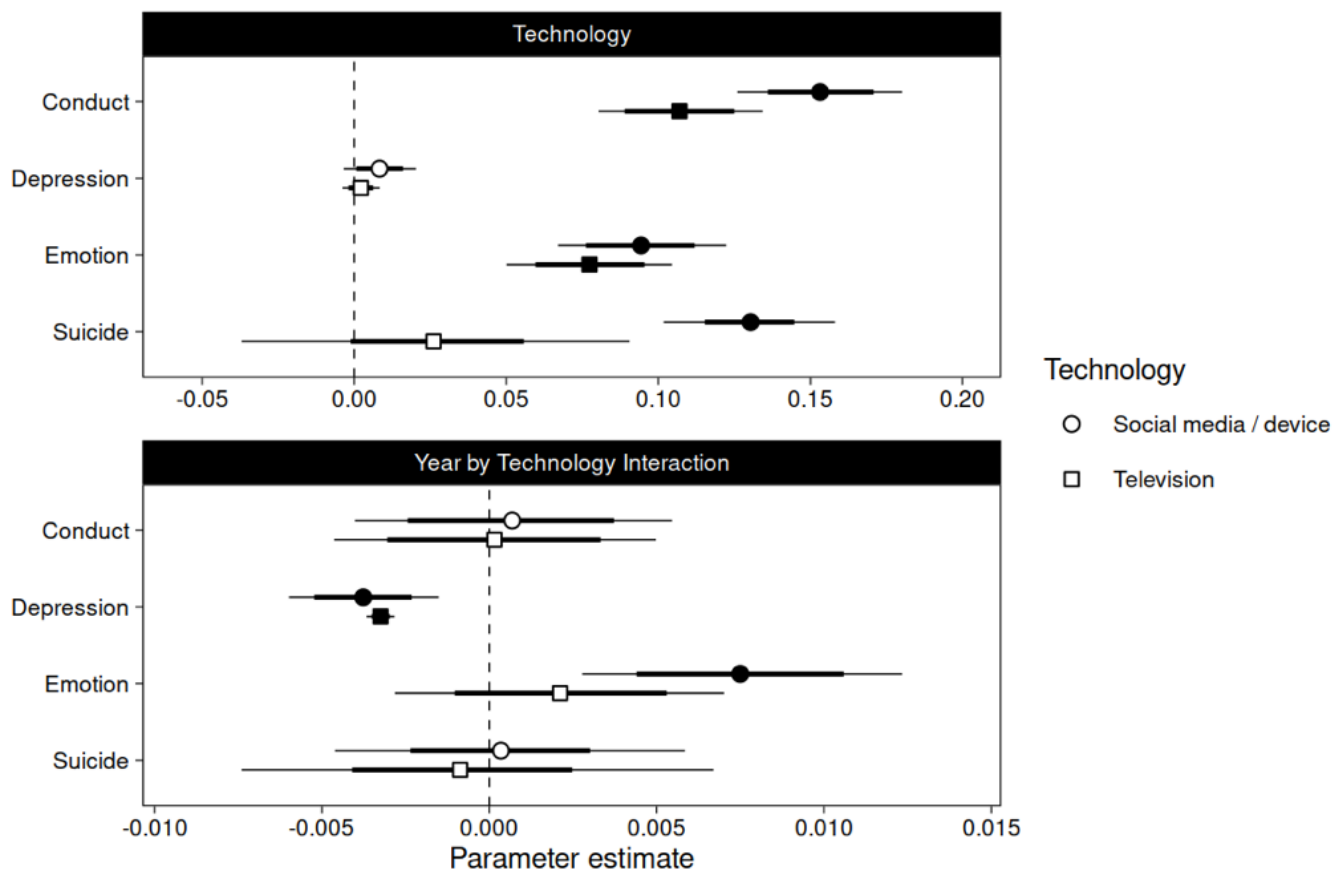

Figure 2. Parameter estimates, in standard deviation units, from models of technology use - mental health associations described in the main text. Top panel: Contributions of technology use on each of the mental health outcomes (y-axis) for the different technology engagement indicators, in 2017. Thick and thin lines indicate $80 \%$ and $95 \%$ CIs, respectively. Filled points indicate parameters whose $95 \% \mathrm{CI}$ excludes zero. Bottom panel: Magnitudes of change in the technology use - mental health associations per one year.

We also investigated whether changes in technology's relations with mental health described in the bottom panel of Figure 2 were moderated by sex, but none of the estimates were credibly different from zero, suggesting that any changes — or lack thereof-over time were not different between boys and girls. We further investigated the robustness of our results with respect to analysis strategy by estimating analogous structural equation models where each mental health outcome was treated as a latent variable indicated by the items discussed above (Rosseel, 2012). Those analyses replicated the above results, with the only exception that the 2017 TV - suicide association's 95\% CI just excluded zero ([0.001, 0.026]; see online analysis supplement). Overall, there was very little evidence for an increase in the negative associations between technology and mental health over time, and some evidence to the contrary.

\section{Relaxing the linearity assumptions}

In our focal analyses we assumed linear relations between technology use and mental health and assumed that any changes to those relations would be linear. Although useful for testing overall decreases/increases, these assump- tions may not be tenable. Specifically, the former assumption might be inaccurate because relations between technology use and mental health are sometimes better described by a quadratic function (Przybylski \& Weinstein, 2017). The latter assumption, in turn, may be too restrictive and thereby miss more complicated patterns of change over time (see Figure 1). Although those assumptions were required to test our critical questions, we therefore conducted additional analyses that eschewed these assumptions.

We estimated two generalized additive models (GAM) of the relation between technology use and mental health using penalized cubic regression splines (Wood, 2017, 2020). The first model included year and technology use as smooth predictors using splines. These splines allowed the associations to take any form indicated by the data without a priori restricting them to e.g. a linear or quadratic form. The second model was identical but included a smooth tensor product interaction between year and technology use, thus allowing the (potentially nonlinear) technology use-mental health association to change (potentially nonlinearly) over time. Because these models allow for more complex patterns, they will necessarily capture a greater proportion of variability in the mental health outcomes than the linear models presented 
above, and therefore provide a useful metric of variance explained in the outcomes. On the other hand, because of the potential complexity of the associations, there is no single parameter that describes the resulting associations, and therefore testing for overall decreases/increases is not straightforward in this framework.
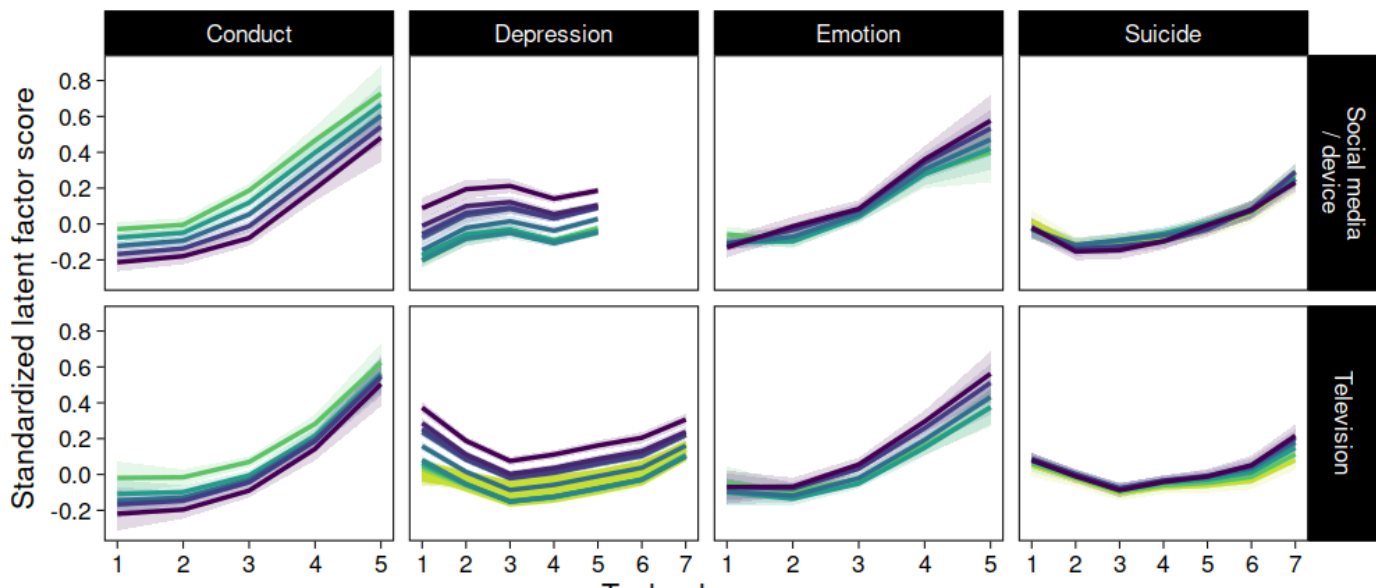

Year

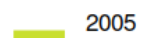

(or before)

2008
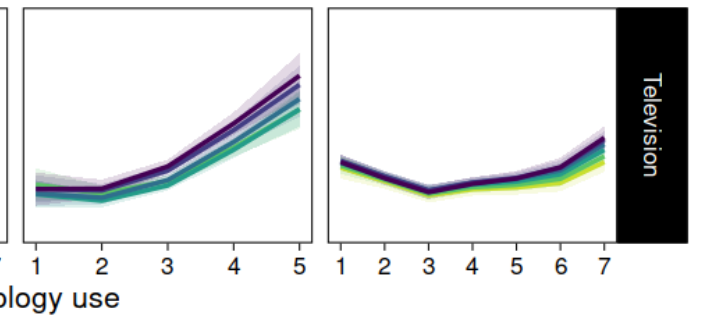

2011

2014
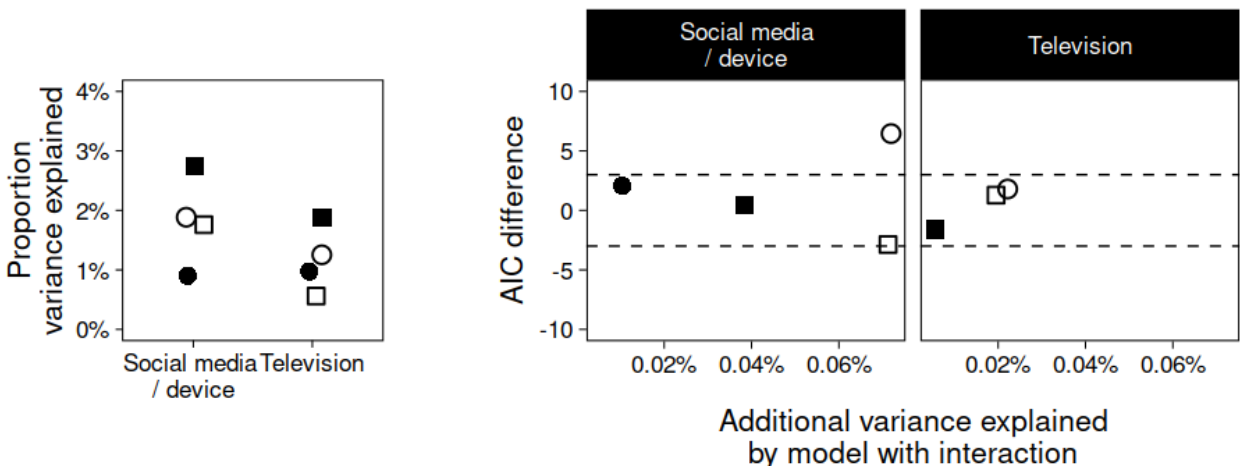

Outcome

- Conduct

- Depression

O Emotion

$\square$ Suicide

by model with interaction

Figure 3. Results from nonlinear Generalized Additive Models of the associations between technology use and mental health. Top panel: Estimated year-specific regression lines (with 95\%CIs as shades) for the relationship between each technology use variable (two rows) and mental health outcome (columns). Bottom left: Proportion of variance explained by the models with year and technology use as nonlinear predictors (but not their interaction). Points are horizontally jittered to display overlapping values. Bottom right: The Akaike Information Criteria difference in favour of the model that also included a nonlinear interaction between year and technology use (y-axis), and the additional proportion of variance explained by that model (x-axis). AIC differences greater than 3 (dashed horizontal lines) indicate better model fit for the model including the interaction term (and smaller than -3 indicate better fit for the non-interactive model); values in between indicate inconclusive differences. Not displayed is the Television-Depression model's point, because its high AIC value (90; additional variance explained $=0.03 \%$ ) rendered the rest of the figure unintelligible.

As can be seen in Figure 3 (top panel), the technology use-mental health relations were complex and generally curvilinear. However, in line with the previous linear models, the GAMs that included year and technology use, but not their interaction, still only explained less than $3 \%$ of variance in the outcomes (Figure 3, bottom left). Critically, most models indicated that the (nonlinear) technology-mental health relationships had not changed over time as suggested by model comparison using AIC (Figure 3, bottom right): There were only two associations, between social media use and emotional problems, and between television viewing and depression, that had changed in potentially nonlinear ways according to the GAM model comparisons (Figure 3, bottom right). The association between TV viewing and depression had generally become slightly more prominently an inverted U-shaped function over time, whereas the association between social media use and emotional problems had become a slightly more linear trend over time (Figure 3, top panel). In sum, even after relaxing assumptions about linearity - in both the relations between technology use and 
mental health, and how they have changed over time - there was little evidence to suggest that there has been drastic change in the associations over time.

\section{Discussion}

In the present study, we investigated the idea that the associations relating adolescent technology engagement with mental health are dynamic and might shift over time. We studied this question by modelling four different mental health outcomes against three forms of technology use across three large nationally representative datasets across 9 to 26 years. From these eight models, we found one clinically relevant self-reported mental health outcome, depression, for which the links to technology use had become consistently less negative over time. However, this decline was found for both television and social media, so we did not obtain strong evidence that the relation between television watching and negative mental health outcomes was unique in waning over long periods of time. Further, we only found one case whereby social media's relation with mental health had become more negative over time: The argument that fast-paced changes to social media platforms and devices have made them more harmful for adolescent mental health in the past decade is, therefore, not strongly supported by current data either.

To investigate the relationships' change over time, we first studied the technology associations specific to 2017, and found that technology uses were weakly associated with higher levels of most mental health problems, apart from depression. In context, because the large sample sizes are likely to make even small relations' confidence intervals exclude zero, interpreting the magnitudes in practical terms might be preferred instead. Previous studies have shown that, in the case of subjective well-being scales, effects of about a half standard deviation are required for people to subjectively experience change (Anvari \& Lakens, 2019). In those terms, our parameter estimates that ranged from zero to about 0.15 , are not likely to indicate practically meaningful associations with mental health. An additional view to the sizes of these associations is afforded by a more detailed look at the analyses of the YRBS data, which contained binary outcomes. For example, males using an average amount of digital devices reported planning for suicide with a $9 \%$ probability, and males who were 1 standard deviation above the mean on device use endorsed the item with a $11 \%$ probability, leading to a small difference $(2 \%, 95 \% \mathrm{CI}$ $[1.6 \%, 2.7 \%])$.

Most importantly to the present investigation, there was no consistent change over time in technology's relations with mental health. Technology associations with conduct problems and suicidality were relatively stable over time. Social media's relations with emotional problems had slightly increased, but television's had not. Unexpectedly, both social media's and television's associations with depression, on the other hand, had decreased. The magnitudes of the observed changes over time were small: In all three cases where parameters' uncertainty intervals excluded zero, the relations were smaller than 0.01 standard deviation units per year, showing that if effects are changing over time, this change is better characterized as a slow drift rather than a rapid shift. In the context of technology use-mental health associations that ranged between zero and 0.15 in 2017, a yearly linear change of 0.01 is unlikely to translate to meaningful changes in associations outside the statistical model. Finally, we did not find any differences in the time courses between girls and boys. Overall, the ideas that technologies we no longer worry about are becoming less harmful or that technologies we worry the most about now are becoming more harmful were not supported in the data we analysed.

Nevertheless, the observed patterns of change - the increased association between social media use and emotional problems, and decreased association between technology engagement and depression - hint at the changing roles of emerging technologies in young people's lives and underlines the scientific value of considering a more dynamic picture of their associations with mental health. Changes in the ways in which technologies are used, such as whether they are used passively or actively (Verduyn et al., 2017), and changes in the features inherent in them (Lewis, 2017; Solon, 2017), could both underlie observed changes in the technologies' associations with mental health. It is possible, then, that the technological advances in social media platforms could be interrelated with their use as part of social support seeking and emotional coping processes (Boyd, 2014). However, because the observed patterns in the current study were contradictory to each other, and not conceptually replicated across the other related outcomes, we advise caution in making strong inferences based on these observations.

More broadly, what might be responsible for the mixed results with respect to changes in technology's relations with mental health? In the context of older technologies, such as $\mathrm{TV}$, our knowledge of social media and digital device use is necessarily limited by their comparatively brief existence. Therefore, our results may partly reflect the shorter observation window of social media and digital device use, in comparison to TV: As is observed in Figure 1, there was an initial long period of relative invariance in TV's relation to mental health, and it is possible that the stability of social media effects observed here reflect such an early period of 
invariance. As more data accumulates on adolescents' use of emerging technologies, our knowledge of them and their effects on mental health will become more precise. It may, then, be premature to draw firm conclusions about the increasing, or declining, associations between social media and adolescent mental health.

We acknowledge additional limitations that prevent firm conclusions based on the data studied here. First, we studied the between-person relationships between technology use and mental health. We partially replicated previous findings whereby individuals who use more technology are likely to report higher mental health problems, but unlike longitudinal within-person data, these findings do not directly afford a causal claim that technology use is responsible for variation in mental health. In fact, some longitudinal studies have found the opposite, whereby mental health problems predict smartphone use, rather than phone use predicting mental health problems (Bado et al., 2020), or found bidirectional relationships (Orben et al., 2019). For example, changes, if any, in associations between technology use and mental health could occur if adolescents with underlying problems were less likely to use established technologies and media. In that case, the initially negative non-causal relation between technology use would become attenuated over time.

In addition, it is important to note that this work, along with previous literature on this topic, to some extent risks conflating between- and within-person associations and causal mechanisms. Although we have discussed associations between technology use and mental health at the level of individuals, it is possible that there are additional or alternative routes of association at other levels of analysis. It is possible, for example, that as a given technology becomes adopted by most individuals in a group, even individuals who do not use that technology could become indirectly impacted by it, either through its impacts on peers, or for example by them being deprived of a novel communication platform where social life now takes place. This type of relationship between technology adoption and mental health might not be apparent in a correlation between individuals' use of technology and mental health and highlights the importance of carefully considering the different implications of hypothesized relations at the potentially different levels of analyses.

We also did not include covariates in our models for two reasons. First, the selection of covariates should be guided by a strong theory that specifies the causal relations between variables (Rohrer, 2018). Currently, no good candidates for such a theory exist, and it was not our purpose here to offer one. It is likely that if meaningful covariates were added, the results might change, but in the absence of a theory those changes would be difficult to understand and might simply reflect inherent variation between models (Orben \& Przybylski, 2019a). Nevertheless, previous studies have found that covariates tend to render related associations smaller (Orben \& Przybylski, 2019b), suggesting that the magnitudes we report are overestimates of the true associations. Second, because our investigation spanned three heterogeneous data sets, it would have been difficult to include covariates while at the same time maintain similarity and parsimony across models. With that in mind, we studied the simplest relations between technology use and mental health, controlling only for the included covariates (gender and year, and their interactions). In light of this, the exact magnitudes of the relations observed here are unlikely to provide accurate estimates of any underlying causal relations between technology use and mental health, if any exist. Going forward, information about how the technologies are used and in which contexts would be required for meaningful causal analyses of the relations, because we would expect that e.g. using devices for staying in touch with relatives would relate to mental health differently than if said devices were used for non-social purposes. Therefore, future work should focus on identifying competing theoretical models that could be tested against data, and collecting sufficiently detailed data to allow those tests.

In addition to the complexities in determining causal relations, the self-report nature of the current data presents unique problems. Although self-report measures of mental health and technology use have high face validity, they might be poor representations of the actual state of affairs (Shaw et al., 2020): In studies of this topic, self-report measures of technology use are consistently biased and are not highly correlated with objective use (Johannes et al., 2020; Parry et al., 2020; Scharkow, 2016). Additionally, true changes in technologies and their associations with health could be difficult to observe in between-persons data because adolescents, too, might change and e.g. adapt to be better able to cope with more harmful technologies.

In light of this, more robust studies of the changing roles of emerging technologies in young people's lives should use data that affords a more detailed, more accurate, and less biased look into the individuals engaging with the technologies. This detail should include both an increased timeframe to be considered and a more fine-grained look at engagement, but also information on how exactly the engagement is taking place beyond, for example, the distinction between active and passive use. Fortunately, such data already exist in the confines of the technology companies behind the most popular online platforms and products. Collaborative work 
between industry-based scientists working for gaming, social media, and technology companies and independent academic scientists would, from this perspective, be greatly beneficial to studying how technologies are affecting individuals and societies.

Concerns that technology is becoming both more prevalent in young people's lives and likewise more harmful to their mental health, has gained traction in recent years. If supported by empirical study, this idea would potentially suggest policy intervention (Department for Digital, Culture, Media \& Sport, 2020). However, we studied changes in adolescent technology use's relation to mental health, and found that, overall, there was little to no evidence showing that technology is becoming more negatively associated with mental health over time. Although we, among others who have examined this topic, studied relations between people, rather than the more appropriate study of change within an individual, our results suggest that just as the ways in which young people use technology is changing, so too may its relations with mental health change, but this change is not well characterized as technology becoming either better or worse. As more data is accrued by global technology stakeholders, we hope that transparent and robust science would emerge in collaboration with those stakeholders in order to better elucidate the changing roles of technology in young people's lives.

\section{Author contributions}

Conceptualization: Matti Vuorre, Amy Orben and Andrew K. Przybylski.

Formal Analysis: Matti Vuorre.

Funding Acquisition: Andrew K. Przybylski.

Investigation: Matti Vuorre, Amy Orben and Andrew K. Przybylski.

Methodology: Matti Vuorre, Amy Orben and Andrew K. Przybylski.

Resources: Andrew K. Przybylski.

Software: Matti Vuorre.

Supervision: Andrew K. Przybylski.

Visualization: Matti Vuorre.

Writing - Original Draft Preparation: Matti Vuorre, Amy Orben and Andrew K. Przybylski.

Writing - Review \& Editing: Matti Vuorre, Amy Orben and Andrew K. Przybylski.

\section{References}

Anderson, M., \& Jiang, J. (2018). Teens' social media habits and experiences. Pew Research Center, 28.

Anvari, F., \& Lakens, D. (2019). Using Anchor-Based Methods to Determine the Smallest Effect Size of Interest. PsyArXiv. https://doi.org/10.31234/osf.io/syp5a
Appel, M., Marker, C., \& Gnambs, T. (2020). Are Social Media Ruining Our Lives? A Review of Meta-Analytic Evidence. Review of General Psychology, 24(1), 60-74. https://doi.org/10.1177/1089268019880891

Bado, P., Schafer, J., Simioni, A. R., Bressan, R. A., Gadelha, A., Pan, P. M., Miguel, E. C., Rohde, L. A., \& Salum, G. (2020). Screen time and psychopathology: Investigating directionality using cross-lagged panel models [Preprint]. https://doi.org/10.31234/osf.io/q8tur

Bjerre-Nielsen, A., Andersen, A., Minor, K., \& Lassen, D. D. (2020). The Negative Effect of Smartphone Use on Academic Performance May Be Overestimated: Evidence From a 2-Year Panel Study. Psychological Science, 0956797620956613.

https://doi.org/10.1177/0956797620956613

Boyd, D. (2014). It's Complicated: The Social Lives of Networked Teens. Yale University Press.

Bürkner, P.-C. (2017). brms: An R Package for Bayesian Multilevel Models Using Stan. Journal of Statistical Software, 80(1), 1-28. https://doi.org/10.18637/jss.v080.i01

Bushman, B. J., \& Anderson, C. A. (2002). Violent Video Games and Hostile Expectations: A Test of the General Aggression Model. Personality and Social Psychology Bulletin, 28(12), 1679-1686. https://oi.org/10.1177/014616702237649

Carpenter, B., Gelman, A., Hoffman, M. D., Lee, D., Goodrich, B., Betancourt, M., Brubaker, M., Guo, J., Li, P., \& Riddell, A. (2017). Stan: A Probabilistic Programming Language. Journal of Statistical Software, 76(1). https://doi.org/10.18637/jss.v076.i01

Centers for Disease Control and Prevention. (2020). WISQARS Fatal Injury Reports. https://webappa.cdc.gov/sasweb/ncipc/mortrate.html

Department for Digital, Culture, Media \& Sport. (2020). Online Harms White Paper: Full Government Response to the consultation. https://www.gov.uk/government/consultations/online-harms-white-paper

Dickson, K., Richardson, M., Kwan, I., MacDowall, W., Burchett, H., Stansfield, C., Brunton, G., Sutcliffe, K., \& Thomas, J. (2019). Screen-based activities and children and young people's mental health and psychosocial wellbeing: A systematic map of reviews.

Drummond, A., Sauer, J. D., \& Ferguson, C. J. (2020). Do longitudinal studies support long-term relationships between aggressive game play and youth aggressive behaviour? A meta-analytic examination. Royal Society Open Science, 7(7), 200373. https://doi.org/10.1098/rsos.200373

Etchells, P. J., Gage, S. H., Rutherford, A. D., \& Munafò, M. R. (2016). Prospective Investigation of Video Game Use in Children and Subsequent Conduct Disorder and 
Depression Using Data from the Avon Longitudinal Study of Parents and Children. PLOS ONE, 11(1), e0147732. https://doi.org/10.1371/journal.pone.0147732

Ferguson, C. J. (2020). Links between screen use and depressive symptoms in adolescents over 16 years: Is there evidence for increased harm? Developmental Science. https://doi.org/10.1111/desc.13008

Ferguson, C. J., Copenhaver, A., \& Markey, P. (2020). Reexamining the Findings of the American Psychological Association's 2015 Task Force on Violent Media: A Meta-Analysis. Perspectives on Psychological Science, 15(6), 1423-1443. https://doi.org/10.1177/1745691620927666

Furedi, F. (2015). The media's first moral panic. History Today, 65(11), 46-8.

Gelman, A., \& Hill, J. (2007). Data Analysis Using Regression and Multilevel/Hierarchical Models. Cambridge University Press.

Goodman, R., Ford, T., Simmons, H., Gatward, R., \& Meltzer, H. (2000). Using the Strengths and Difficulties Questionnaire (SDQ) to screen for child psychiatric disorders in a community sample. The British Journal of Psychiatry, 177(6), 534-539. https://doi.org/10.1192/bjp.177.6.534

Grimes, T., Anderson, J. A., \& Bergen, L. (2008). Media Violence and Aggression: Science and Ideology. SAGE.

Hawkes, N. (2019). CMO report is unable to shed light on impact of screen time and social media on children's health. $B M J, 364$. https://doi.org/10.1136/bmj.1643

Jensen, M., George, M. J., Russell, M. R., \& Odgers, C. L. (2019). Young Adolescents' Digital Technology Use and Mental Health Symptoms: Little Evidence of Longitudinal or Daily Linkages. Clinical Psychological Science, 7(6), 1416-1433. https://doi.org/10.1177/2167702619859336

Johannes, N., Vuorre, M., \& Przybylski, A. K. (2020). Video game play is positively correlated with well-being. PsyArXiv. https://doi.org/10.31234/osf.io/qrjza

Kann, L. (2018). Youth Risk Behavior SurveillanceUnited States, 2017. MMWR. Surveillance Summaries, 67. https://doi.org/10.15585/mmwr.ss6708a1

Kardefelt-Winther, D., Rees, G., \& Livingstone, S. (2020). Contextualising the link between adolescents' use of digital technology and their mental health: A multi-country study of time spent online and life satisfaction. Journal of Child Psychology and Psychiatry, jcpp.13280. https://doi.org/10.1111/jcpp.13280

Kelly, Y., Zilanawala, A., Booker, C., \& Sacker, A. (2018). Social Media Use and Adolescent Mental Health: Findings From the UK Millennium Cohort Study. EClinicalMedicine, $6, \quad 59-68$. https://doi.org/10.1016/j.eclinm.2018.12.005

Kreski, N., Platt, J., Rutherford, C., Olfson, M., Odgers, C., Schulenberg, J., \& Keyes, K. M. (2020). Social Media Use and Depressive Symptoms Among United States Adolescents. Journal of Adolescent Health, S1054139X20304031.

https://doi.org/10.1016/j.jadohealth.2020.07.006

Lewis, P. (2017, October 6). 'Our minds can be hijacked': The tech insiders who fear a smartphone dystopia. The Guardian. https://www.theguardian.com/technology/2017/oct/05/smartphone-addiction-silicon-valleydystopia

Miech, R. A., Johnston, L. D., O’Malley, P. M., Bachman, J. G., Schulenberg, J. E., \& Patrick, M., E. (2020). Monitoring the Future national survey results on drug use, 1975-2019: Volume I, Secondary school students. The University of Michigan. http://monitoringthefuture.org/pubs.html

Newcomb, M. D., Huba, G. J., \& Bentler, P. M. (1981). A Multidimensional Assessment of Stressful Life Events among Adolescents: Derivation and Correlates. Journal of Health and Social Behavior, 22(4), 400-415. JSTOR. https://doi.org/10.2307/2136681

Odgers, C. L., \& Jensen, M. R. (2020). Annual Research Review: Adolescent mental health in the digital age: facts, fears, and future directions. Journal of Child Psychology and Psychiatry, 61(3), 336-348. https://doi.org/10.1111/jcpp.13190

Ophir, Y., Lipshits-Braziler, Y., \& Rosenberg, H. (2020). New-Media Screen Time Is Not (Necessarily) Linked to Depression: Comments on Twenge, Joiner, Rogers, and Martin (2018). Clinical Psychological Science, 8(2), 374378. https://doi.org/10.1177/2167702619849412

Orben, A. (2020). The Sisyphean Cycle of Technology Panics. Perspectives on Psychological Science, 1745691620919372.

https://doi.org/10.1177/1745691620919372

Orben, A., Dienlin, T., \& Przybylski, A. K. (2019). Social media's enduring effect on adolescent life satisfaction. Proceedings of the National Academy of Sciences, 116(21), 10226-10228. https://doi.org/10.1073/pnas.1902058116

Orben, A., \& Przybylski, A. K. (2019a). The association between adolescent well-being and digital technology use. Nature Human Behaviour, 3(2), 173-182. https://doi.org/10.1038/s41562-018-0506-1

Orben, A., \& Przybylski, A. K. (2019b). Screens, Teens, and Psychological Well-Being: Evidence From Three TimeUse-Diary Studies. Psychological Science, 30(5), 682696. https://doi.org/10.1177/0956797619830329

Parry, D. A., Davidson, B. I., Sewall, C., Fisher, J. T., Mieczkowski, H., \& Quintana, D. (2020). Measurement Discrepancies Between Logged and Self-Reported Digital Media Use: A Systematic Review and Meta-Analysis. https://doi.org/10.31234/osf.io/f6xvz 
Preston, M. I. (1941). Children's reactions to movie horrors and radiocrime. The Journal of Pediatrics, 19(2), 147168. https://doi.org/10.1016/S0022-3476(41)80059-6

Przybylski, A. K., \& Weinstein, N. (2017). A Large-Scale Test of the Goldilocks Hypothesis: Quantifying the Relations Between Digital-Screen Use and the Mental WellBeing of Adolescents. Psychological Science, 28(2), 204215. https://doi.org/10.1177/0956797616678438

Rohrer, J. M. (2018). Thinking Clearly About Correlations and Causation: Graphical Causal Models for Observational Data. Advances in Methods and Practices in Psychological

Science. https://doi.org/10.1177/2515245917745629

Rosseel, Y. (2012). lavaan: An R Package for Structural Equation Modeling. Journal of Statistical Software, 48(1), 1-36. https://doi.org/10.18637/jss.v048.i02

Scharkow, M. (2016). The Accuracy of Self-Reported Internet Use-A Validation Study Using Client Log Data. Communication Methods and Measures, 10(1), 13-27. https://doi.org/10.1080/19312458.2015.1118446

Shaw, H., Ellis, D. A., Geyer, K., Davidson, B. I., Ziegler, F. V., \& Smith, A. (2020). Quantifying smartphone 'use': Choice of measurement impacts relationships between 'usage' and health [Preprint]. PsyArXiv. https://doi.org/10.31234/osf.io/mpxra

Singer, N. (2016, May 14). When Websites Won't Take No for an Answer (Published 2016). The New York Times. https://www.nytimes.com/2016/05/15/technology/personaltech/when-websites-wont-take-no-for-an-answer.html

Solon, O. (2017, November 9). Ex-Facebook president Sean Parker: Site made to exploit human 'vulnerability'. The Guardian. https://www.theguardian.com/technology/2017/nov/09/facebook-sean-parker-vulnerabilitybrain-psychology

Taylor, K., \& Silver, L. (2019). Smartphone ownership is growing rapidly around the world, but not always equally. Pew Research Center, 5.

Twenge, J. M., Joiner, T. E., Rogers, M. L., \& Martin, G. N. (2017). Increases in Depressive Symptoms, Suicide-Related Outcomes, and Suicide Rates Among U.S. Adolescents After 2010 and Links to Increased New Media Screen Time. Clinical Psychological Science, 2167702617723376. https://doi.org/10.1177/2167702617723376

University of Essex, Institute for Social and Economic Research. (2019). Understanding Society: Waves 1-9, 20092018 and Harmonised BHPS: Waves 1-18, 1991-2009. [Data collection]. 12th Edition. UK Data Service. http://doi.org/10.5255/UKDA-SN-6614-13
Verduyn, P., Ybarra, O., Résibois, M., Jonides, J., \& Kross, E. (2017). Do Social Network Sites Enhance or Undermine Subjective Well-Being? A Critical Review: Do Social Network Sites Enhance or Undermine Subjective Well-Being? Social Issues and Policy Review, 11(1), 274302. https://doi.org/10.1111/sipr.12033

Viner, R., Davie, M., \& Firth, A. (2019). The health impacts of screen time: A guide for clinicians and parents. Edinburgh, Scotland: Royal College of Paediatrics and Child Health.

Wertham, F. (1954). Seduction of the innocent. Rinehart New York.

Weston, S. J., Ritchie, S. J., Rohrer, J. M., \& Przybylski, A. K. (2019). Recommendations for Increasing the Transparency of Analysis of Preexisting Data Sets: Advances in Methods and Practices in Psychological Science. https://doi.org/10.1177/2515245919848684

Wood, S. N. (2017). Generalized Additive Models: An Introduction with R, Second Edition. CRC Press.

Wood, S. N. (2020). mgcv: Mixed GAM Computation Vehicle with Automatic Smoothness Estimation (1.8-33) [Computer software]. https://CRAN.R-project.org/package $=\mathrm{mgcv}$ 